\title{
Esterifications in Ionic Liquids with 1-Alkyl-3-Methylimidazolium Cation and Hydrogen Sulfate Anion: Conversion and Phase Equilibrium
}

\author{
Deyan Naydenov $^{\mathrm{a}}$, Hans Hasse ${ }^{\mathrm{b}}$, Gerd Maurer $^{\mathrm{b}}$ and Hans-Jörg Bart*,a,c \\ ${ }^{a}$ Lehrstuhl für Thermische Verfahrenstechnik, TU Kaiserslautern; ${ }^{b}$ Lehrstuhl für Thermodynamik, TU Kaiserslautern; \\ ${ }^{c}$ Center of Mathematical and Computational Modeling, TU Kaiserslautern, Postfach 3049, D-67653 Kaiserslautern, \\ Germany
}

\begin{abstract}
The esterification of ethanol, 1-propanol and 1-butanol with acetic acid in three ionic liquids with $\mathrm{HSO}_{4}{ }^{-}$anion and 1-alkyl-3-methylimidazolium cation was investigated. The ionic liquids are 1-methylimidazolium hydrogen sulfate $[\mathrm{HMIM}]\left[\mathrm{HSO}_{4}\right]$, 1-ethyl-3-methylimidazolium hydrogen sulfate [EMIM] $\left[\mathrm{HSO}_{4}\right]$ and 1-butyl-3-methylimidazolium hydrogen sulfate $[\mathrm{BMIM}]\left[\mathrm{HSO}_{4}\right]$, which have catalytic activity. Data and modeling on the reaction conversions and the distribution of the compounds between the phases is reported here. Trends for the change in the liquid-liquid equilibrium, with parameters like alkyl chain length on the cation or the alcohol, are discussed and used to estimate the phase behavior of similar esterification systems.
\end{abstract}

\section{INTRODUCTION}

Esterifications are industrially important reactions and have been widely studied. In the recent years there are a number of publications concerning the use of ionic liquids (IL) as catalyst and/or solvent for esterification reactions [121]. It was stated that IL can be beneficial compounds for these reactions, reducing the need for volatile solvents and allowing easier separation of the ester and catalyst $[2,21]$. In the majority of published literature the IL used exhibited Brønsted acidity and were used as both catalyst and solvent in bulk quantities. Typical examples are IL with $\mathrm{HSO}_{4}{ }^{-}$or $\mathrm{H}_{2} \mathrm{PO}_{4}{ }^{-}$anion, otherwise the IL were only solvents and an additional catalyst $[4,5]$ was added (e.g. p-toluenesulfonic acid). In most cases the miscibility of the used IL with the corresponding esters is reported, when the IL were used in bulk quantities. The upper phase contained most of the ester and the lower phase the ionic liquid and most of the water. This phase splitting is beneficial for the separation of the ester and offers the opportunity to shift the equilibrium conversion towards products as reported in literature [2, 7, 16]. However, often the effect of the ionic liquid on the conversion shift is not quite clear, since no comparison was made with the homogeneous reaction without it. In few cases no phase separation was observed, as the IL used was miscible with esters. The latter cases were not suited for biphasic operation, as even the addition of water did not induce phase splitting [15].

The distribution of the compounds between the phases or more detailed information on the phase equilibrium is scarcely mentioned [7,15], although it is expected to be important for achieving higher conversions. In general there are few publications on liquid-liquid equilibria for systems with ionic liquids and it is nearly impossible to conclude a

*Address correspondence to this author at the Center of Mathematical and Computational Modeling, TU Kaiserslautern, Postfach 3049, D-67653 Kaiserslautern, Germany; E-mail: bart@mv.uni-kl.de priori whether an IL is suited for a given esterification reaction. Besides the effect on reaction conversion, the phase equilibrium will be important for processing the products of the reaction. Since the reaction is seldom completed in one stage the unreacted alcohol and acid need to be separated. Also, the loss of IL, due to solubility in the organic product phase, is usually neglected.

We already published [22] the phase equilibria for ternary systems containing products/reactants of the esterifica-tion reaction of ethanol with acetic acid and several ionic liquids at $40{ }^{\circ} \mathrm{C}$. Furthermore, we investigated the effect of the alcohol/ester on the LLE in ternary systems [23]. In this paper we expand our study to five component systems. The focus is on the conversion of esterification reactions in ionic liquids with differently substituted methylimidazolium cation and hydrogen sulfate anion and the distribution of the compounds between the two phases for the five component systems containing alcohol + acetic acid + ester + water + IL.

alcohol $(\mathrm{ROH})+\operatorname{acid}\left(\mathrm{CH}_{3} \mathrm{COOH}\right) \leftrightarrow \operatorname{ester}\left(\mathrm{CH}_{3} \mathrm{COOR}\right)+$ $\mathrm{H}_{2} \mathrm{O}$

The investigated ionic liquids are 1-methylimidazolium hydrogen sulfate [HMIM] $\left[\mathrm{HSO}_{4}\right]$, 1-ethyl-3-methylimidazolium hydrogen sulfate [EMIM] $\left[\mathrm{HSO}_{4}\right]$ and 1-butyl-3methylimidazolium hydrogen sulfate $[\mathrm{BMIM}]\left[\mathrm{HSO}_{4}\right]$. Also in the experiments the alkyl chain on the alcohol is varied (esterification of ethanol, 1-propanol and 1-butanol with acetic acid). The change in phase equilibrium is discussed and the obtained trends are used to make a rough estimate of the behavior of systems with higher alcohols. Combining the results with previous studies the relationship between reaction conversion and phase equilibrium is discussed and remarks on the choice of ILs for esterifications of aliphatic alcohols with acids in ionic liquids are made.

\section{EXPERIMENTAL}

The ionic liquids $[\mathrm{EMIM}]\left[\mathrm{HSO}_{4}\right]$ and $[\mathrm{BMIM}]\left[\mathrm{HSO}_{4}\right]$ were provided by BASF with a purity of $\geq 95 \%$ and were 
Table 1. Literature Values of the Equilibrium Ratio $K_{x}$ and the Corresponding Equilibrium Conversions

\begin{tabular}{|c|c|c|c|c|}
\hline System & Ref. & $\mathbf{K x}$ & Conversion & Temperature \\
\hline 1-Propylacetate & $\begin{array}{l}{[28]} \\
{[24]}\end{array}$ & $\begin{array}{c}4.9 \\
4.07\end{array}$ & $\begin{array}{l}68.9 \% \\
66.9 \%\end{array}$ & $\begin{array}{l}80^{\circ} \mathrm{C}^{* *} \\
155^{\circ} \mathrm{C}\end{array}$ \\
\hline 1-Butylacetate & $\begin{array}{c}{[24]} \\
{[5]}\end{array}$ & $\begin{array}{c}4.24 \\
-\end{array}$ & $\begin{array}{l}67.3 \% \\
69.2 \%\end{array}$ & $\begin{array}{c}155^{\circ} \mathrm{C} \\
60^{\circ} \mathrm{C}\end{array}$ \\
\hline
\end{tabular}

"-not dependent on temperature, ${ }^{* *}$-extrapolated.

used without further purification. The ionic liquid $[\mathrm{HMIM}]\left[\mathrm{HSO}_{4}\right]$ was synthesized from 1-methylimidazole (> $99.5 \%)$ and sulfuric acid. Its purity was estimated to be 98 $\%$ [22]. The water content of the ionic liquids was usually less than $0.1 \%$. Otherwise the initial water quantity is indicated in the Tables 2a to 4a. Ethanol (99.8 \%), npropanol (99.8\%), n-butanol (99.5\%), acetic acid (99.8\%), n-propylacetate $(98 \%)$, n-butylacetate $(99.5 \%)$ and methanol $(99.8 \%)$ were used without additional purification.

The desired amounts of the alcohol, acetic acid and the ionic liquid were placed in a $100 \mathrm{ml}$ flask, which was immersed in a water (or oil) bath at the desired temperature $(\mathrm{T} \pm 0.1 \mathrm{~K})$. The reaction mixture was mechanically stirred. Although some of the (alcohol + IL) systems reveal miscibility gap at room temperature, all starting mixtures were homogeneous at the temperature of the reaction experiments $\left(60{ }^{\circ} \mathrm{C}\right.$ or $\left.80{ }^{\circ} \mathrm{C}\right)$. After some time, depending on the system (usually between $15 \mathrm{~min}$ and 2 hours), the formation of a second liquid phase was observed. Methanol was added (used as internal standard) to the organic phase sample, and it was analyzed by GC to determine the volatile compounds (alcohol, acetic acid and ester). The organic phase, when placed in the GC vial at room temperature, usually becomes milky and methanol also acts as a modifier to yield a single phase. Water (in both phases) was measured by Karl-Fischer titration. Acetic acid and the IL (in both phases) were determined by titration with $0.1 \mathrm{M} \mathrm{NaOH}$. The reaction conversion was calculated from the water and/or acetic acid content. For this purpose the mass of both phases had to be determined as well. For some preliminary experiments only the water content of the phases was determined and used to calculate the conversion. Since the alcohol and the ester in the IL were not directly measured, their concentration was calculated by a mass balance. Due to experimental uncertainties the calculated values for alcohol and ester in the IL have a high relative error (approx. 10-20 $\%$ ). When these concentrations are low, e.g. below 0.05 mass fractions, the relative error can be much higher. However, the knowledge of alcohol and ester in the ionic phase is, in any case, useful when calculating the reaction conversion.

\section{RESULTS AND DISCUSSION}

Esterifications are relatively well studied reactions. Typical values of the equilibrium ratio $K_{x}=x_{\text {ester }} \cdot x_{\text {water }}$ t $\left(x_{\text {alcohol }} \cdot x_{\text {acid }}\right)$ found in the literature for the investigated reactions using conventional catalysts, e.g. $\mathrm{H}_{2} \mathrm{SO}_{4}$, are presented in Table 1 . These values are very close and the theoretically obtained conversions are in the range of 66-69 $\%$ (except [5] and [29], see Table 1). It is generally stated that these esterifications have negligible heat of reaction and the $K_{x}$ is, therefore, almost independent of the temperature for all reactions.

The experimental conditions and the conversions, based on the reaction limiting reactant, for the three reactions are listed in Tables $2 \mathbf{a}, 3 \mathbf{a}$ and $4 \mathbf{a}$. The composition of the phases in mass fractions is presented in Tables $\mathbf{2 b}, \mathbf{3 b}$ and $\mathbf{4 b}$. The results for the esterification of acetic acid with ethanol at $60^{\circ} \mathrm{C}$ are presented in Tables $\mathbf{2 a}$ and $\mathbf{2} \mathbf{b}$, with n-propanol in Tables 3a and 3b and with n-butanol in Tables $\mathbf{4 a}$ and $\mathbf{4 b}$. The cation ([HMIM], [EMIM], [BMIM]) and the alcohol was varied (ethanol, n-propanol and n-butanol) allowing to study the effect of the alkyl chain length. The reaction temperatures were chosen so that they are below the boiling point of the lowest boiling compound at atmospheric pressure and are $60^{\circ} \mathrm{C}$ for the ethylacetate and $80^{\circ} \mathrm{C}$ for the propylacetate and butylacetate systems. In a typical experiment the mass of the ionic liquid is about $1 / 3$ of the overall mass, higher or lower IL quantities showed only a weak influence on the conversion in the studied range. Reaction times were mostly kept at $24 \mathrm{~h}$ to achieve both phase and reaction equilibrium in all cases. These reaction times are high compared to the ones reported in the literature for other IL and esterification systems, though it is clear that often even 8 to $12 \mathrm{~h}$ would be sufficient (see e.g. Table 3a). The obtained results indicate that these IL act as weak catalysts. For shorter reaction times in an industrial application the addition of e.g. $\mathrm{H}_{2} \mathrm{SO}_{4}$, would be helpful.

It was found, that in some cases, particularly when the alcohol is better soluble in the ionic phase, it may react with the $\mathrm{HSO}_{4}{ }^{-}$anion to produce an alkylsulfate anion:

$\mathrm{ROH}+\mathrm{HSO}_{4}{ }^{-} \leftrightarrow \mathrm{RSO}_{4}{ }^{-}+\mathrm{H}_{2} \mathrm{O}$

This reaction is accelerated by higher temperatures and free acids like $\mathrm{H}_{2} \mathrm{SO}_{4}$. Its presence is indicated by the higher quantities of produced water in comparison to the consumed acetic acid. This side reaction is not mentioned so far in the available literature on esterifications in $\mathrm{IL}_{\text {with }} \mathrm{HSO}_{4}{ }^{-}$anion.

\section{Variation of the Cation of the IL}

The $[\mathrm{HMIM}]\left[\mathrm{HSO}_{4}\right]$ systems exhibited about $10 \%$ increased conversion compared to homogeneous systems without IL and was the best of all IL being investigated. 
Table 2a. Esterification of Acetic Acid with Ethanol in Different Ionic Liquids at $\mathbf{T}=\mathbf{6 0}^{\circ} \mathrm{C}$ : Initial Conditions

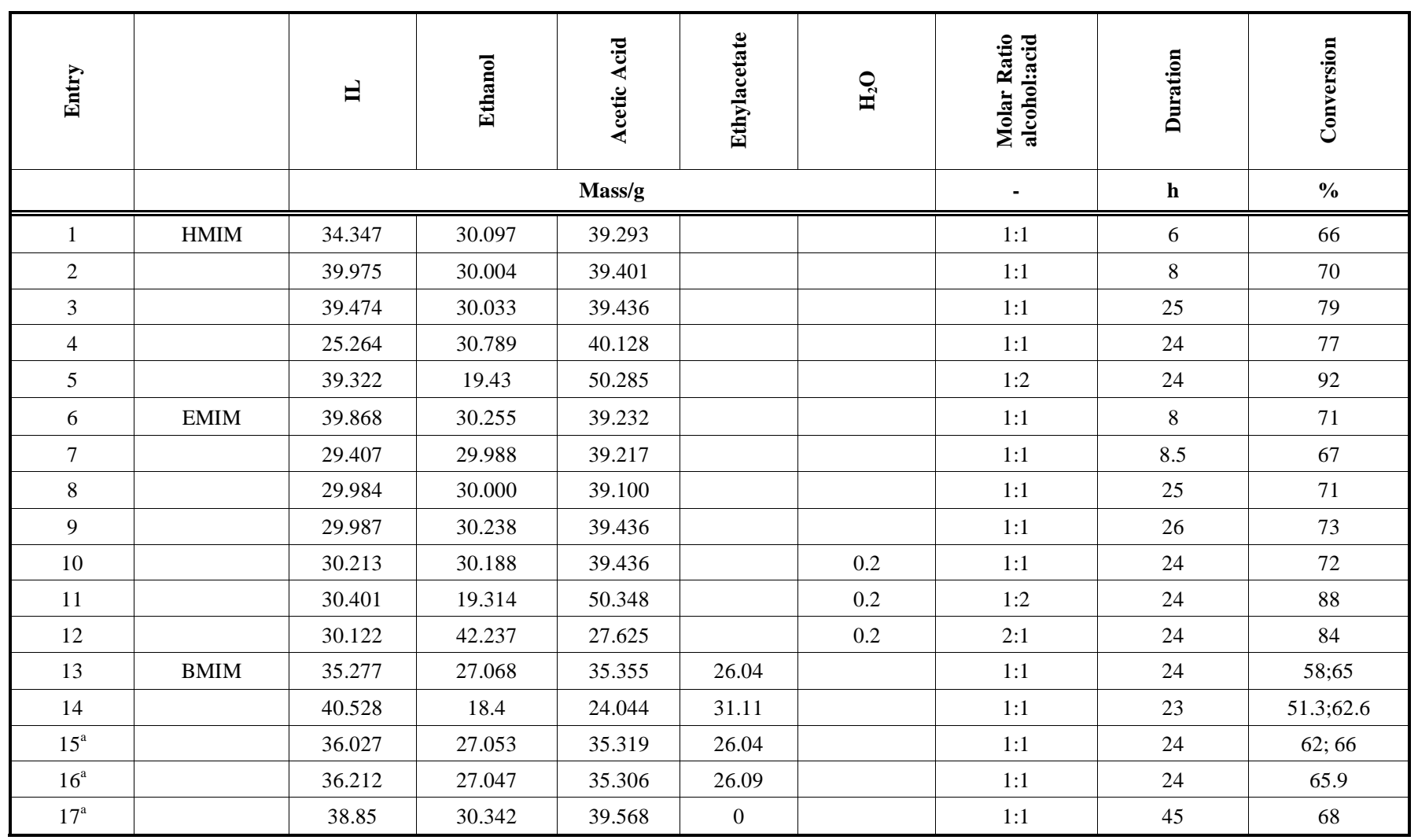

a -at room temperature $25^{\circ} \mathrm{C}$.

Table 2b. Esterification of Acetic Acid with Ethanol in Different Ionic Liquids at $\mathrm{T}=60^{\circ} \mathrm{C}$ : Composition of the Coexisting Phases (in Mass Fractions)

\begin{tabular}{|c|c|c|c|c|c|c|c|c|c|c|c|}
\hline \multirow[t]{2}{*}{ Entry } & & \multicolumn{5}{|c|}{ Organic Phase } & \multicolumn{5}{|c|}{ Ionic Phase } \\
\hline & & $\underset{\text { 空 }}{\stackrel{\Xi}{\Xi}}$ & 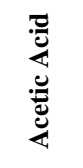 & 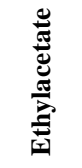 & $\begin{array}{l}\underset{N}{\beth} \\
\underset{I}{\mid c}\end{array}$ & $\exists$ & 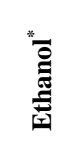 & 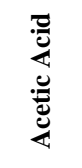 & 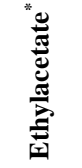 & ્ְతి & $\exists$ \\
\hline 1 & HMIM & & & & 0.0326 & & & & & 0.1252 & \\
\hline 2 & & & & & 0.0283 & & & & & 0.1243 & \\
\hline 3 & & 0.076 & 0.101 & 0.799 & 0.0249 & 0 & 0.043 & 0.058 & 0.086 & 0.148 & 0.71 \\
\hline 4 & & 0.098 & 0.118 & 0.747 & 0.038 & 0.005 & 0.04 & 0.069 & 0.057 & 0.189 & 0.66 \\
\hline 5 & & 0.026 & 0.31 & 0.67 & 0.0264 & $<0.001$ & 0.005 & 0.214 & 0.078 & 0.0944 & 0.6 \\
\hline 6 & EMIM & 0.119 & 0.098 & 0.752 & 0.0275 & & 0.058 & 0.114 & 0.108 & 0.1172 & \\
\hline 7 & & & & & 0.0345 & & & & & 0.1310 & \\
\hline 8 & & & & & 0.0347 & & & & & 0.1450 & \\
\hline 9 & & & 0.114 & & 0.0355 & & & 0.113 & & 0.15 & \\
\hline 10 & & 0.101 & 0.112 & 0.746 & 0.0346 & 0.009 & 0.063 & 0.112 & 0.089 & 0.151 & 0.607 \\
\hline 11 & & 0.03 & 0.297 & 0.62 & 0.0365 & 0.051 & 0.022 & 0.295 & 0.225 & 0.0845 & 0.396 \\
\hline 12 & & 0.291 & 0.052 & 0.58 & 0.0447 & 0.061 & 0.196 & 0.044 & 0.088 & 0.111 & 0.554 \\
\hline 13 & BMIM & 0.089 & 0.106 & 0.71 & 0.0337 & 0.079 & 0.092 & 0.127 & 0.437 & 0.0605 & 0.288 \\
\hline 14 & & 0.062 & 0.075 & 0.858 & 0.015 & 0.024 & 0.083 & 0.119 & 0.330 & 0.0495 & 0.390 \\
\hline $15^{\mathrm{a}}$ & & 0.083 & 0.091 & 0.78 & 0.0235 & 0.02 & 0.082 & 0.123 & 0.267 & 0.0855 & 0.520 \\
\hline $16^{\mathrm{a}}$ & & 0.076 & 0.085 & 0.794 & 0.020 & 0.012 & 0.072 & 0.113 & 0.221 & 0.0902 & 0.570 \\
\hline $17^{\mathrm{a}}$ & & 0.094 & 0.102 & 0.751 & 0.0284 & 0.029 & 0.089 & 0.127 & 0.258 & 0.0899 & 0.468 \\
\hline
\end{tabular}

"-calculated by mass balance.

at room temperature $25^{\circ} \mathrm{C}$. 
Table 3a. Esterification of Acetic Acid with Propanol in Different Ionic Liquids at $\mathbf{T}=\mathbf{8 0}^{\circ} \mathrm{C}$ : Initial Conditions

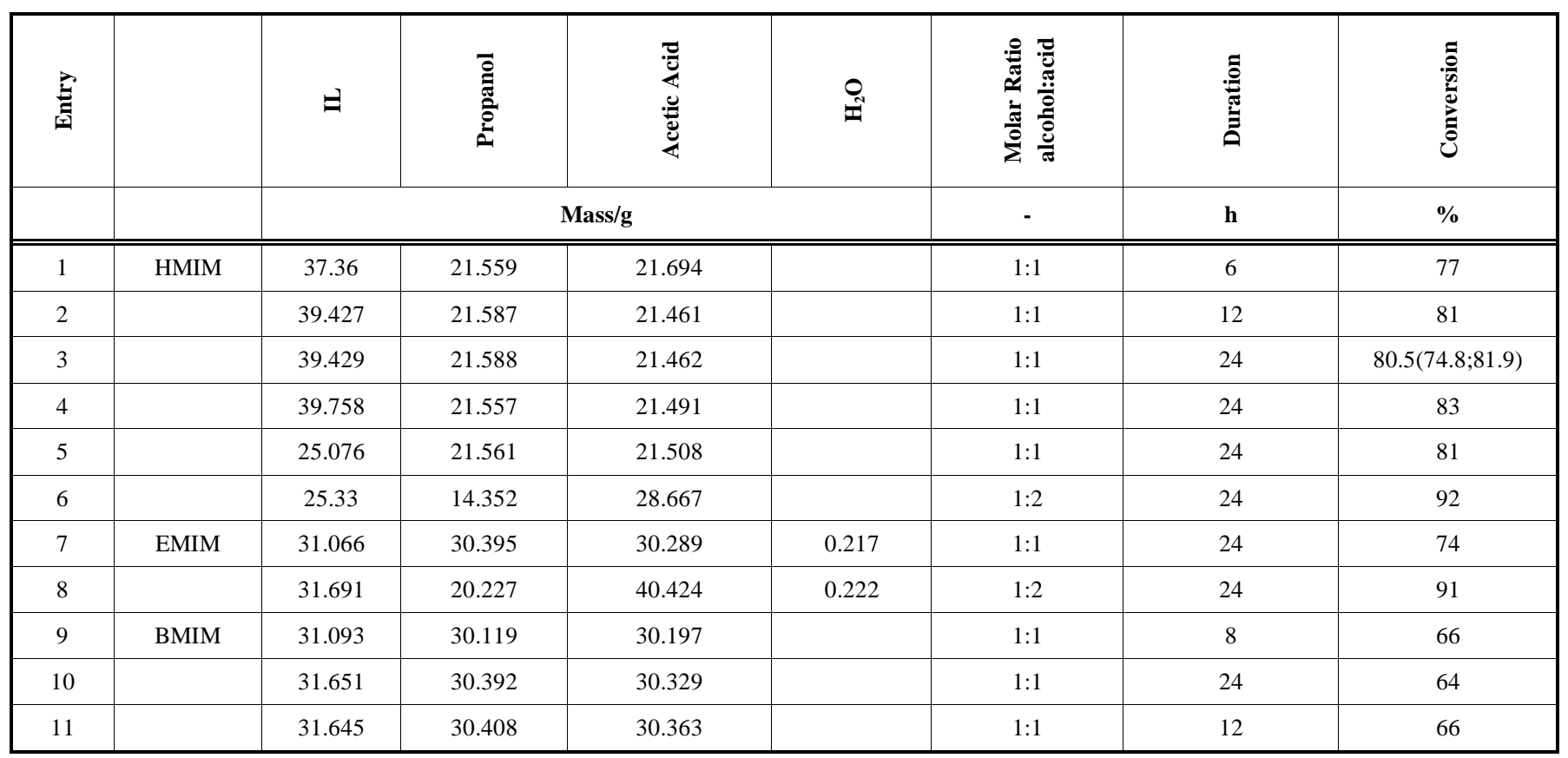

Table 3b. Esterification of Acetic Acid with Propanol in Different Ionic Liquids at $\mathrm{T}=\mathbf{8 0}^{\circ} \mathrm{C}$ : Composition of the Coexisting Phases (in Mass Fractions)

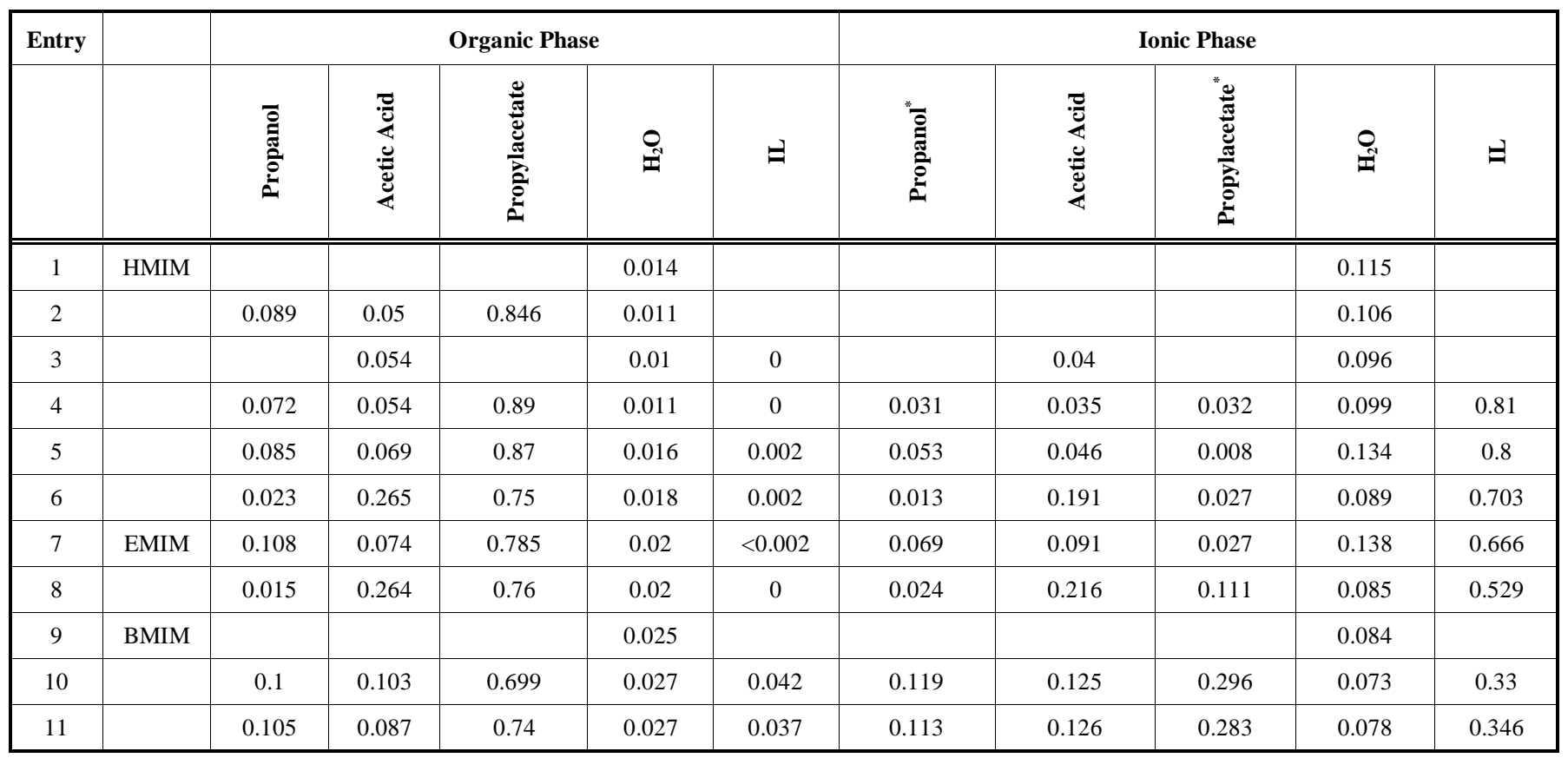

"-calculated by mass balance.

Similar to the results obtained for the ternary systems [22] we also observe the largest miscibility gaps here. This is verified by the fact that the mutual solubilities of ester and $\mathrm{IL}$ are low and that for the [HMIM] $\left[\mathrm{HSO}_{4}\right]$ systems the formation of a relatively small amount of the ester through reaction already leads to phase splitting. The most of the formed ester (85-90\%) is found in the organic phase, since its solubility in the ionic phase is lower, compared to the other IL systems. This is, as we believe, the main reason for the better results achieved with this IL. Both ethanol and acetic acid, are somewhat better soluble in the organic phase than in the ionic phase, which was expected, taking into account the ternary systems with [HMIM] $\left[\mathrm{HSO}_{4}\right]$ [22]. Typical for this IL is the slightly lower distribution ratio of water between the two phases, $D_{H 2 O}=w^{O P} / w^{I L}$ compared to the other systems. When less IL is used (e.g. entry 4 in Table 2a) only slightly lower conversion is achieved. Thus, there is a potential for optimizing the IL quantity and lowering the 
Table 4a. Esterification of Acetic Acid with Butanol in Different Ionic Liquids at $\mathbf{T}=\mathbf{8 0}{ }^{\circ} \mathrm{C}$ : Initial Conditions

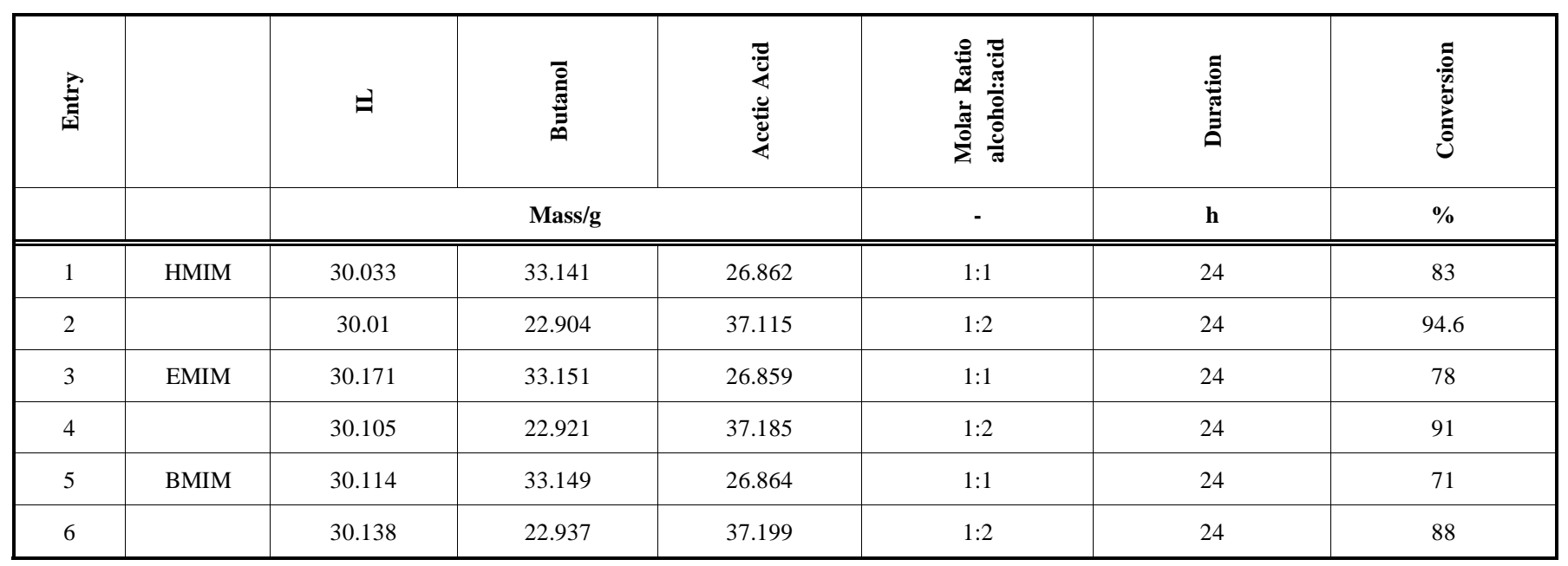

Table 4b. Esterification of Acetic Acid with Butanol in Different Ionic Liquids at $\mathbf{T}=80^{\circ} \mathrm{C}$ : Composition of the Coexisting Phases (in Mass Fractions)

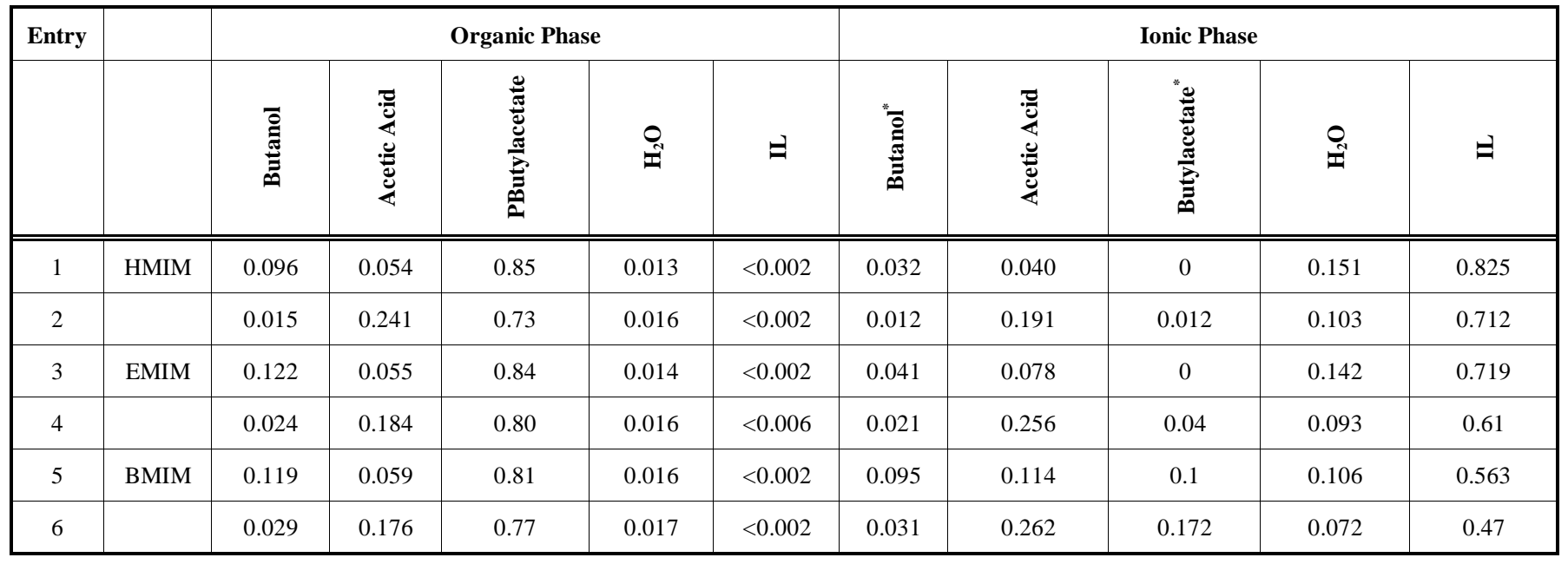

"-calculated by mass balance.

costs. A two-fold excess of acetic acid can shift the conversion to $92 \%$ of the alcohol (an improvement of ca. 7 $\%$ compared to the case without IL) at the expense of higher purification costs.

Lower conversion shifts were achieved for the $[\mathrm{EMIM}]\left[\mathrm{HSO}_{4}\right]$ systems, compared to the $[\mathrm{HMIM}]\left[\mathrm{HSO}_{4}\right]$. The ethanol conversion is 72 to $73 \%$ (about $5-6 \%$ shift compared to systems without IL) compared to about $78 \%$ conversion (corresponding to ca. 10-11 \% shift) with $[\mathrm{HMIM}]\left[\mathrm{HSO}_{4}\right]$. A surplus of acetic acid to ethanol $(2: 1)$ gave $88 \%$ conversion, which is hardly any improvement to the case without IL. With a ratio of ethanol to acetic acid of 2:1 only $84 \%$ conversion was achieved, attributed to the side reaction. Any nonstoechiometrical ratio of the reactants typically leads to significantly higher solubilities of the IL in the organic phase. While there is only $0.9 \%$ EMIM in the ester phase (Table $\mathbf{2 b}$, entry 10), about 5-6 \% IL are found for ratios lower or higher than unity (entries 11 and 12 (1:2 and 2:1)). Interestingly the distribution coefficient of acetic acid between the two phases is near unity, and (entry 12 in Table 2b) it is slightly better soluble in the organic phase than in the IL. This was not expected, as already published elsewhere [22], acetic acid in the ternary system acetic acid + ethylacetate $+[\mathrm{EMIM}]\left[\mathrm{HSO}_{4}\right]$ is better soluble in the IL at $40{ }^{\circ} \mathrm{C}$.

The $[\mathrm{BMIM}]\left[\mathrm{HSO}_{4}\right]$ experiments differ from the HMIM and EMIM systems, here the addition of ethylacetate was needed to induce phase separation at $60^{\circ} \mathrm{C}$. Addition of ester to induce phase separation was not needed for the systems with n-propylacetate and n-butylacetate. Due to the very small two-phase region for the $[\mathrm{BMIM}]\left[\mathrm{HSO}_{4}\right]$ systems, there is a significant amount of ester present in the IL (in some cases up to $2 / 3$ of the formed ester is in the IL-phase) ,as well as a high IL amount in the organic phase (up to $8 \%$ BMIM in ethylacetate). The high ester content in the ionic phase is, as we believe, the main reason for the lower conversions achieved with BMIM, compared to the other two IL. At lower temperatures the miscibility gap widens (Table 2a, entry 15 to 17 ) and there was a second phase (entry 17) without addition of ester. The obtained conversion in this case was about $67-68 \%$, corresponding to that without IL. Especially for the experiments with BMIM at $60^{\circ} \mathrm{C}$, it 
was found that in some cases the conversions based on water (63\%) and acetic acid (51\%) differ significantly, which means that there is more water in the system than produced by the esterification reaction. The side reaction is not observed or passes only to a low extent in the cases where the alcohol is better soluble in the ester than in the IL.

The phase behavior of the five component systems corresponds to that experimentally determined for the ternary systems [22]. A decrease in the size of the two phase region with increasing the alkyl chain length on cation is found. HMIM systems have the largest miscibility gaps with the lowest mutual solubility of ester and IL and the reactants are better soluble in the organic phase. For the $[\mathrm{BMIM}]\left[\mathrm{HSO}_{4}\right]+$ ethylacetate systems the two-phase region is so small at $60{ }^{\circ} \mathrm{C}$, that the system remains homogeneous at reaction equilibrium. The distribution of the reactants between the two-phases corresponds to that of the ternary systems, as the reactants are better miscible with the IL than with the ester. Additionally, there is a clearly recognizable trend in the reaction conversion with the cation of the IL. Increasing the alkyl chain length of the cation leads to lower conversion for all three reactions. This may be explained with the better solubility of the ester in the catalytic phase, promoting the back reaction and leading to lower reaction conversions. These observations are in agreement with literature [14], where similar effects are observed for IL with an alkylammonium cation.

\section{Variation of the Alkyl Chain of the Alcohol}

The increase of the alkyl chain length of the alcohol and the ester leads to an increase of the miscibility gap for the ternary systems alcohol + ester + IL and acetic acid + ester + IL [23]. The same is true for the five component reactive systems. For example, as mentioned above, with BMIM (entry 13 in Table $\mathbf{2 a}$ ) the formation of second phase is only observed if ethylacetate is added, with butylacetate (entry 5 in Table 4a) addition was not necessary. As a general rule, the less ester is found in the ionic liquid the longer the alkyl chain of the alcohol is. During the experiments the formation of second phase is observed earlier, compared to the ethanol- ethylacetate systems. As a consequence of this bigger miscibility gap, the biphasic esterification of the higher alcohols (propanol, butanol) is possible at higher concentrations of the reactants. This also allows the operation at a nonstoechiometric feed ratio and the use of less IL to induce the phase separation. Due to the smaller miscibility gaps in the ethanol/ethylacetate systems it is disadvantageous to have nonstoechiometric ratios (particularly with [EMIM] $\left[\mathrm{HSO}_{4}\right]$ and $\left.[\mathrm{BMIM}]\left[\mathrm{HSO}_{4}\right]\right)$, since the mutual solubility of ester and IL may increase significantly. In some cases biphasic operation would be impossible as the operating points may lie outside the immiscibility region. When increasing the alkyl chain length, the solubility of alcohol in the ionic (catalytic) phase is decreased and, for higher alcohols (and esters), this could lead to slower kinetics and minor conversions.

The reaction conversions in one stage were at maximum $10-15 \%$ higher than the values for the homogeneous esterifications without IL. Values over $85 \%$ were obtained only if the reactants were in a nonstochiometrical ratio. Higher conversion $(>90 \%, 1: 1)$ for similar reactions in these ionic liquids may only be expected if they exhibit anyway high $K_{x}$ values. Although all three IL are almost fully immiscible with the studied esters, the size of the two phase region differs markedly with the cation of the IL. Systems with shorter alkyl chain on the cation exhibit sufficiently big miscibility gaps. Biphasic operations with $[\mathrm{BMIM}]\left[\mathrm{HSO}_{4}\right]$ and lower alcohols are only possible in a very limited concentration range. For all systems most of the water formed is found in the IL phase and typical distribution ratios $D_{H 2 O}=w^{O P} / w^{I L}$ are about 0.2 . Generally it is observed that the distribution ratio rises slightly with increasing alkyl chain length on the alcohol/ester and decreases with the alkyl rest on the cation of the IL.

\section{Modeling}

We simulated the reaction conversion using a mathematical model presented elsewhere [30]. It is assumed that the reaction occurs only in the ionic phase. The reaction equilibrium is approximated for all three reactions by an

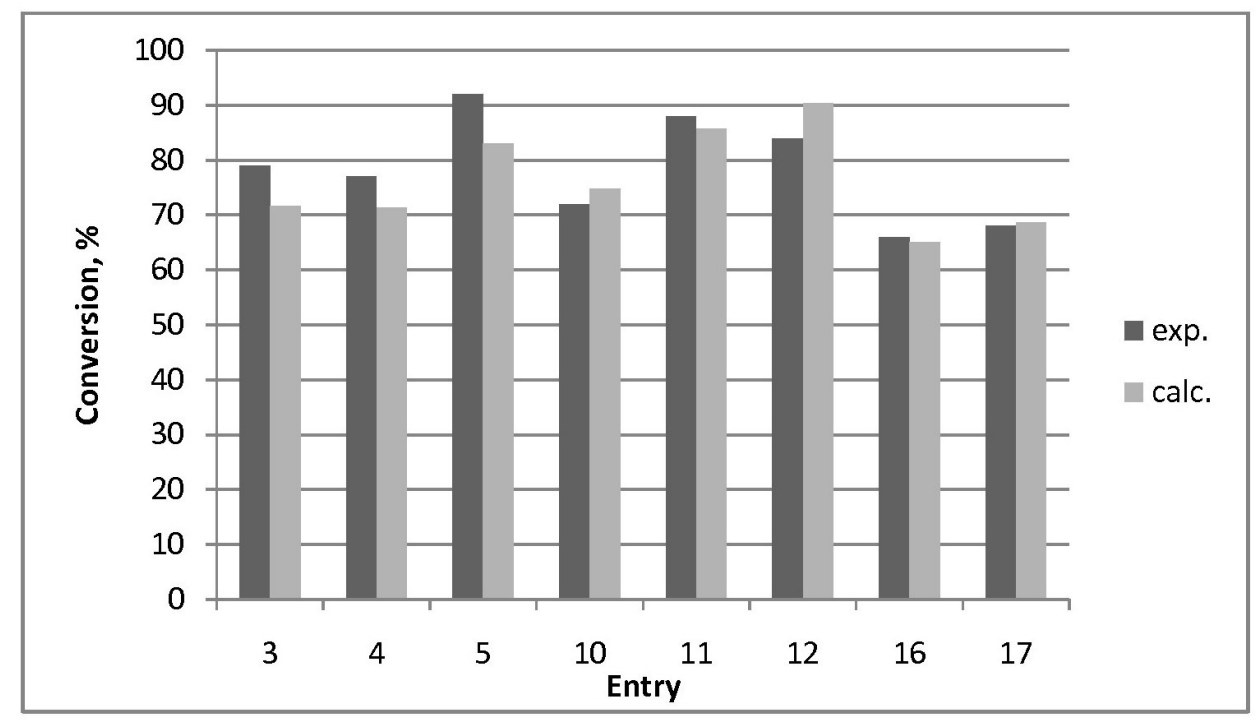

Fig. (1). Comparison of the calculated and experimental reaction conversions for the esterification of ethanol with acetic acid at $60^{\circ} \mathrm{C}$. (s. Tables $\mathbf{2 a}$ and $\mathbf{2 b}$ ). 
equilibrium constant $K_{x}=4$. The side reaction was neglected and the phase equilibrium was approximated by the distribution coefficients obtained from the corresponding experiment. A comparison of the experimental and calculated conversions is presented in Figs. ( $\mathbf{1}$ to $\mathbf{3}$ ). With these assumptions we obtained good results for the esterifications of ethanol and 1-propanol with acetic acid, but high deviations for 1-butanol. It is obvious, however that when the ratio of 1-butanol to acetic acid is 1:2 the match is much better. Also, for the esterifications in BMIM we obtained better compliance than for the other ionic liquids. The highest deviations in the conversion are usually observed when the concentrations of the alcohol and ester in the ionic phase are low. So we suppose that this higher difference is caused by the higher error in the calculation of the alcohol and ester mass fractions giving inaccurate values of the distribution ratio.
Using this model we performed a parameter study, varying the distribution ratios $D=w^{O P} / w^{I L}$ of the compounds to obtain the influence of the phase equilibrium on the conversion. The simulations gave higher conversions when there is a good separation of the products. The more ester removed from the ionic phase (high $D_{\text {ester }}$ values) the better the calculated conversions. Under similar other conditions ( $D_{\text {ester }}, D_{H 2 O}$ and $D_{I L}$ constant) somewhat higher conversions are simulated, if both reactants (alcohol and acid) are better soluble in the catalytic phase $\left(D_{\text {reactant }}<1\right)$ compared to being better soluble in the organic phase $\left(D_{\text {reactant }}>1\right)$. Also the calculated ester content of the organic phase is higher when the reactants are better miscible with the ionic phase, giving higher product purity. If the value of $D_{\text {reactant }}$ is much higher than 1 , this may lead to the decrease of conversion. It is obvious that in this case the reactants are extracted from the

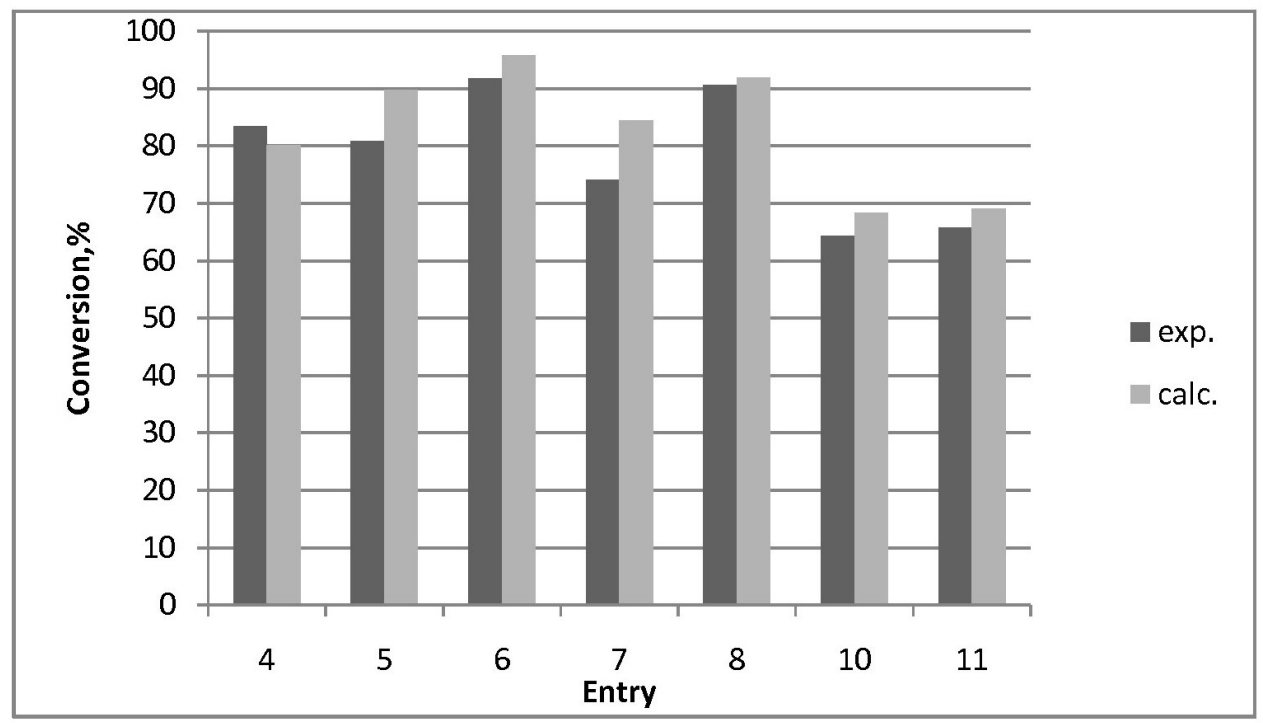

Fig. (2). Comparison of the calculated and experimental reaction conversions for the esterification of 1-propanol with acetic acid at $80^{\circ} \mathrm{C}$. (s. Tables 3a and 3b).

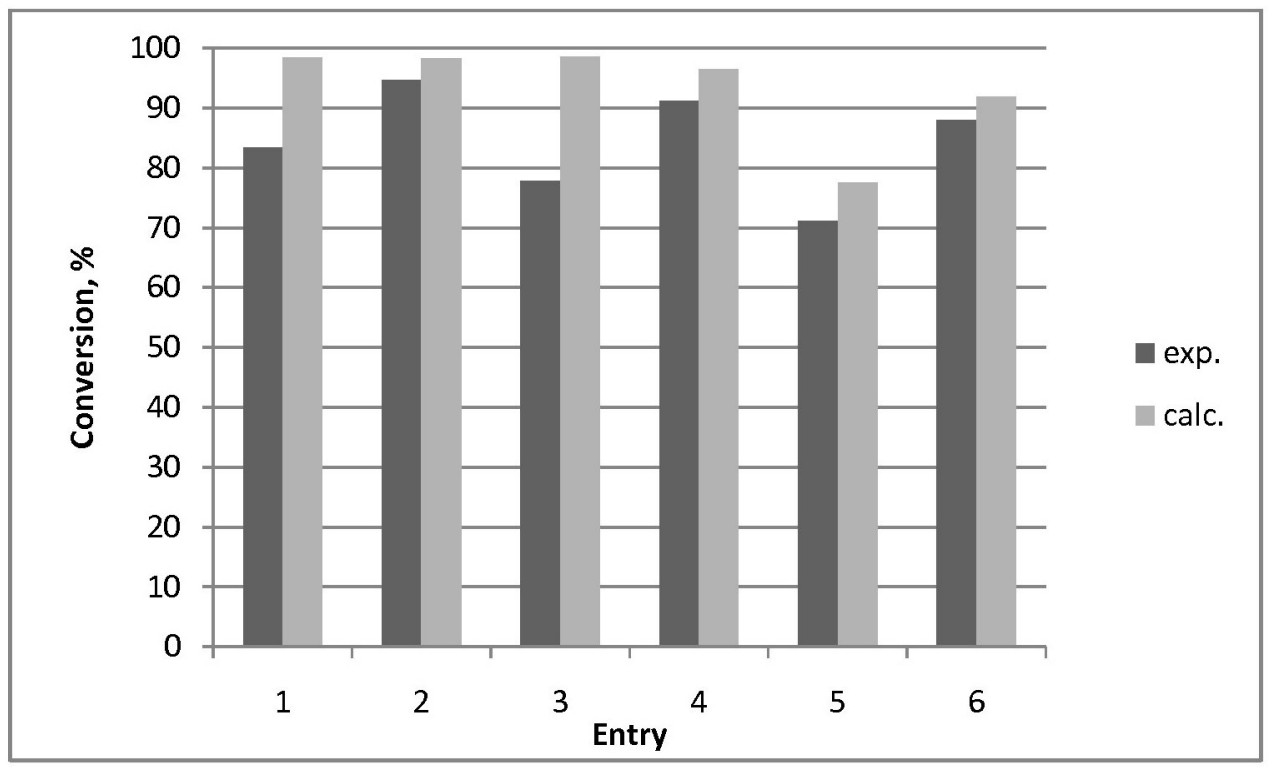

Fig. (3). Comparison of the calculated and experimental reaction conversions for the esterification of 1 -butanol with acetic acid at $80^{\circ} \mathrm{C}$. (s. Tables $\mathbf{4 a}$ and $\mathbf{4 b}$ ). 


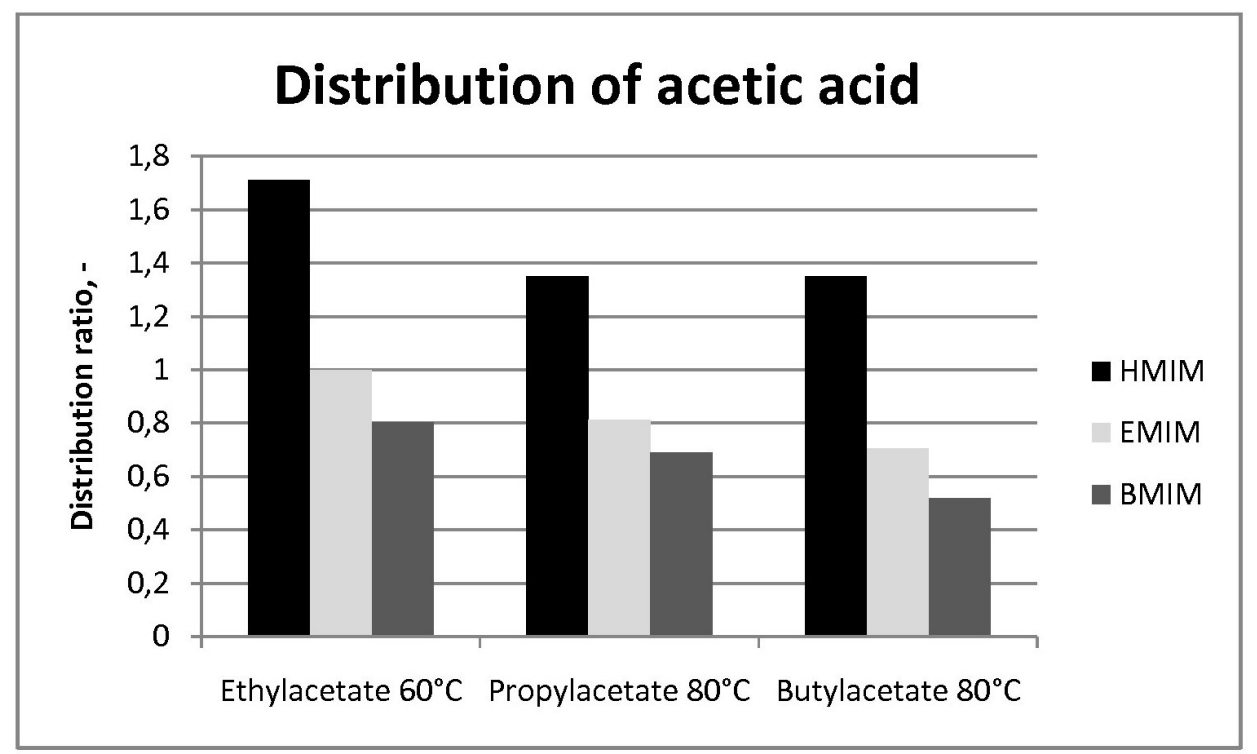

Fig. (4). Distribution ratio $\mathrm{D}=\mathrm{w}^{\mathrm{OP}} / \mathrm{w}^{\mathrm{IL}}$ for acetic acid. The data for esterification of ethanol with acetic acid in BMIM is at room temperature.

ionic (catalytic) phase, which may have a negative effect on the reaction conversion.

\section{SUMMARY}

The miscibility gaps for esterification of lower alcohols with lower acids are small (particularly when BMIM is used), so that the biphasic operation is almost impossible or the addition of ester is necessary to induce phase separation. The two-phase region increases by decreasing the alkyl chain length of the cation and increasing the alkyl chain of the alcohol. Thus, for higher alcohols biphasic operation is always possible using any of the studied IL, also in the cases of a nonstoichiometric feed ratio. The distribution of the reactants between the ester and IL is also dependent on the cation and alkyl chain length of alcohol or acid (See Figs. 4 and 5). Only for the esterification of the lower alcohols (up to propanol) with lower acids (up to acetic acid), one can expect to have one or both reactants in the catalytic (ionic) phase and this only for cations with longer alkyl chain ([EMIM] and [BMIM]). For all other cases we expect to have both the alcohol and the acid in the organic phase with the distribution ratio $D_{\text {reactant }}$ rising when the alkyl chain length of the alcohol or acid is increased.

The results from this study, combined with the experimental data from ternary liquid-liquid equilibria studies presented elsewhere [22, 23], lead to following conclusions in respect to the reaction conversion. For esterifications of aliphatic alcohols with acids, the most decisive parameter is the size of the immiscibility region. As shown, there is a relationship between the reaction conversion and the miscibility gap. A bigger one leads to better separation of the products and higher conversion. As mentioned, when increasing the alkyl rest $\mathrm{R}$ (both of alcohol and acid), it is guaranteed that the immiscibility region will

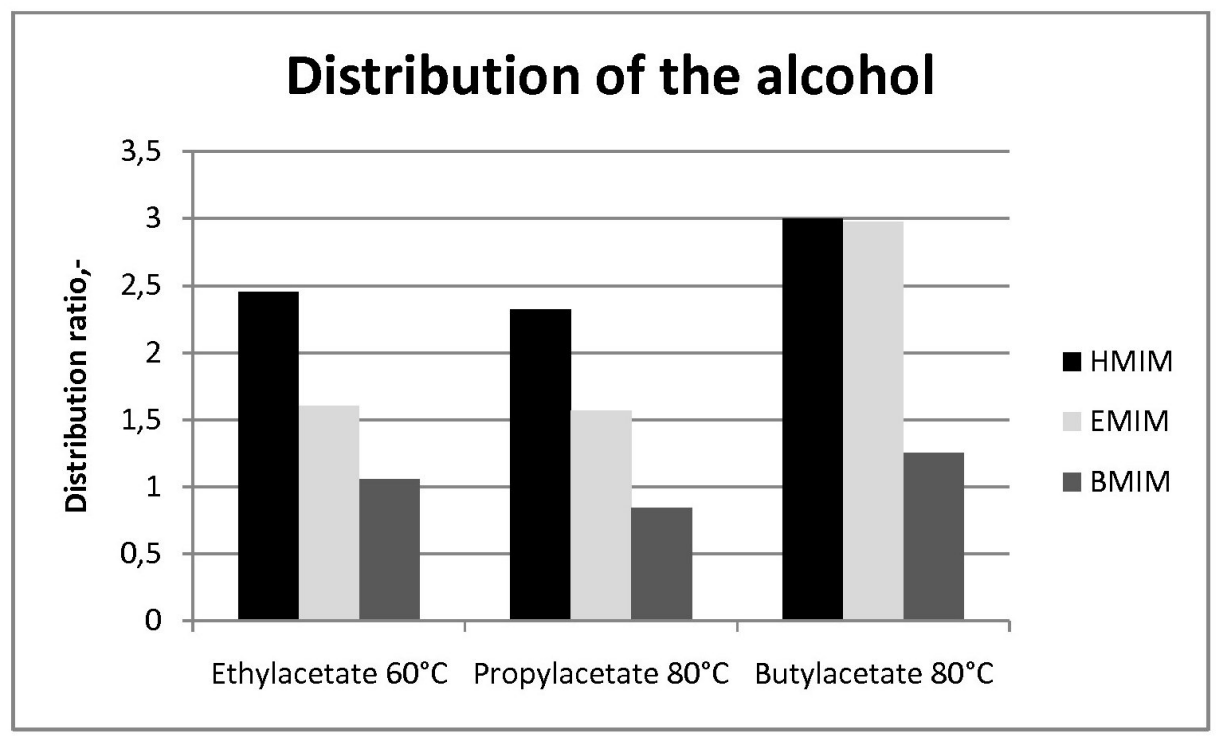

Fig. (5). Distribution ratio $\mathrm{D}=\mathrm{w}^{\mathrm{OP}} / \mathrm{w}^{\mathrm{IL}}$ for ethanol. The data for esterification of ethanol with acetic acid in BMIM is at room temperature. 
rise, having a positive effect on the reaction conversion for the esterification of higher alcohols or acids. A second aspect is the distribution of the reactants between the phases. An unsuitable one (reactants less soluble in the catalytic phase, which will be typical for alcohols higher than butanol and acids higher than butyric acid) may lead to lower conversions or slower kinetics. In such cases it will be better to have the catalyst in the organic phase or a higher mass transfer area will be needed to promote kinetics. The preference is, when a larger miscibility gap is combined with the presence of both reactants in the catalytic phase. As shown, a longer alkyl chain of the cation improves the solubility of the reactants in the IL but leads to a smaller miscibility gap. For this reason we will not observe this case for esterifications of aliphatic alcohols with carbon acids using IL with 1-alkyl-3-methylimidazolium cation and hydrogen sulfate anion. This may explain the obtained moderate conversions compared to values reported in literature $[11,13,16,20]$.

\section{CONCLUSIONS}

Although all studied ionic liquids are immiscible with esters, the size of the two-phase region for the systems varies significantly with, e.g. the cation of the IL. Thus, from the immiscibility of the IL with esters it cannot be simply concluded that the system will allow optimal biphasic operation. While the systems with a shorter alkyl chain of the cation (e.g. [HMIM] $\left[\mathrm{HSO}_{4}\right]$ ) generally exhibit bigger miscibility gaps, the systems with $[\mathrm{BMIM}]\left[\mathrm{HSO}_{4}\right]$, especially these with lower alcohols, have very small ones, thus biphasic operation is only possible in a limited concentration range. For all systems, most of the formed water is found in the ionic phase and the formed ester usually generates a second phase. However, depending on the size of the miscibility gap up to $2 / 3$ of the ester may remain in the ionic phase. A better separation of the products leads to better conversion and, for this reason, the systems with bigger two-phase regions ([HMIM] $\left[\mathrm{HSO}_{4}\right]$ ) gave higher reaction conversions than those with a smaller one ([BMIM] $\left.\left[\mathrm{HSO}_{4}\right]\right)$. The obtained conversion shift, in one stage, was not higher than $10-15 \%$ in the best case. Values over $85 \%$ were obtained only if the reactants were used in a nonstoechiometrical ratio. Higher conversions (> 90\%, 1:1) for similar reactions in these ionic liquids can only be expected if anyway higher $K_{x}$ values are involved.

The phase equilibrium (both the size of the two-phase region and the distribution of the compounds between the phases) varies with the cation of the ionic liquid or with the alcohol. Ionic liquids with a longer alkyl chain of the cation are better miscible with one or both reactants, while e.g. for $[\mathrm{HMIM}]\left[\mathrm{HSO}_{4}\right]$ in all studied cases, both the alcohol and the acid were better miscible with the organic phase. The trends in phase equilibrium obtained for the five-compoundsystems agree, in general, with the results from the threecompound-systems. These results allow the estimation of the phase behavior in esterification systems with IL with 1alkyl-3-methylimidazolium cation and hydrogen sulfate anion.

\section{NOTATION}

$D=$ Distribution ratio

$K_{x}=$ Reaction equilibrium ratio defined with mole fractions

$$
\begin{aligned}
& w=\text { mass fraction, } \mathrm{kg} / \mathrm{kg} \\
& x=\text { mole fraction, } \mathrm{mol} / \mathrm{mol} \\
& \mathrm{OP}=\text { Organic Phase } \\
& \mathrm{IL}=\text { Ionic liquid }
\end{aligned}
$$

\section{ACKNOWLEDGEMENTS}

We would like to thank BASF for providing the ionic liquids [EMIM][HSO4] and [BMIM][HSO4]. The research project is supported by "NanoKat" at TU Kaiserslautern and by funds of the Bundesministerium für Wirtschaft and Technologie (BMWi) through the Arbeitsgemeinschaft industrieller Forschungsvereinigungen (AiF), project Nr.14995 N/2. The authors are grateful for the financial support.

\section{REFERENCES}

[1] Y. Deng, F. Shi, J. Beng, and K. Qiao, "Ionic liquid as a green catalytic reaction medium for esterifications", J. Mol. Catal. A, vol. 165, pp. 33-36, 2001.

[2] J. Fraga-Dubreuil, K. Bourahla, M. Rahmouni, J. P. Bazureau, and J. Hamelin, "Catalysed esterifications in room temperature ionic liquids with acidic counteranion as recyclable reaction media", Catal. Commun., vol. 3, pp. 185-190, 2002.

[3] H.-P. Zhu, F. Yang, J. Tang, and M.-Y. He, "Brønsted acidic ionic liquid 1-methylimidazolium tetrafluoroborate: a green catalyst and recyclable medium for esterification", Green Chem., vol. 5, pp. 3839, 2003.

[4] H.-P. Nguyen, S. Znifeche, and M. Baboulène, "An improved greener esterification of fatty alcohols using a renewable acid ionic liquid couple as catalyst - solvent", Synth. Commun., vol. 34, pp. 2085-2093, 2004.

[5] T. Jiang, Y. Chang, G. Zhao, B. Han, and G. Yang, "Effect of ionic liquids on the chemical equilibrium of esterification of carboxylic acids with alcohols", Synth. Commun., vol. 34, pp. 225-230, 2004.

[6] J. Gui, X. Cong, D. Liu, X. Zhang, Z. Hu, and Z. Sun, "Novel Brønsted acidic ionic liquid as efficient and reusable catalyst system for esterification", Catal. Commun., vol. 5, pp. 473-477, 2004

[7] H. Xing, T. Wang, Z. Zhou, and Y. Dai, "Novel Brønsted-acidic ionic liquids for esterifications", Ind. Eng. Chem. Res., vol. 44, pp. 4147-4150, 2005.

[8] T. Joseph, S. Sahoo, and S. B. Halligudi, "Brönsted acidic ionic liquids: a green, efficient and reusable catalyst system and reaction medium for Fischer esterification”, J. Mol. Catal. A, vol. 234, pp. 107-110, 2005 .

[9] P. Izàk, N. Mateus, C. Alfonso, and J. Crespo, "Enhanced esterification conversion in a room temperature ionic liquid by integrated water removal with pervaporation", Sep. Purif. Tech., vol. 41, pp. 141-145, 2005.

[10] Z. Zhang, W. Wu, B. Han, T. Jiang, B. Wang, and Z. Liu, "Phase separation of the reaction system induced by $\mathrm{CO}_{2}$ and conversion enhancement for the esterification of acetic acid with ethanol in ionic liquid", J. Phys. Chem. B, vol. 109, pp. 16176-16179, 2005.

[11] D. Fang, X.-L. Zhou, Z.-W. Ye, and Z.-L. Liu, "Brønsted acidic ionic liquids and their use as dual solvent-catalysts for Fischer esterifications", Ind. Eng. Chem. Res., vol. 45, pp. 7982-7984, 2006.

[12] D. Barahona, P. H. Pfromm, and M. E. Rezac, "Effect of water activity on the lipase catalysed esterification of geraniol in ionic liquid [bmim] $\mathrm{PF}_{6}$ ", Biotech. Bioeng., vol. 93, pp. 318-324, 2006.

[13] H. Zhang, F. Xu, X. Zhou, G. Zhang, and C. Wang, "A Brønsted acidic ionic liquid as an efficient and reusable catalyst system for esterification", Green Chem., vol. 9, pp. 1208-1211, 2007.

[14] P. A. Ganeshpure, G. George, and J. Das, "Application of triethylammonium salts as ionic liquid catalyst and medium for Fischer esterification", ARKIVOC, vol. 8, pp. 273-278, 2007.

[15] P. A. Ganeshpure, G. George, and J. Das, "Brønsted acidic ionic liquids derived from alkylamines as catalysts and mediums for Fischer esterification: Study on structure-activity relationship", $J$. Mol. Catal. A, vol. 279, pp. 182-186, 2008. 
[16] X. Li, and W. Eli, "A green approach for the synthesis of long chain aliphatic acid esters at room temperature", J. Mol. Catal. A, vol. 279, pp. 159-164, 2008.

[17] X. Li, W. Eli, and G. Li, "Solvent-free synthesis of benzoic esters and benzyl esters in novel Brønsted acidic ionic liquids under microwave irradiation", J. Catal. Commun., vol. 9, pp. 2264-2268, 2008.

[18] T. P. Wells, J. Hallett, C. K. Williams, and T. Welton, "Esterifications in ionic liquids: the influence of solvent basicity", J. Org. Chem., vol. 73, pp. 5585-5588, 2008.

[19] L. Gubicza, K. Bélafi-Bakó, E. Fehér, and T. Fráter, "Waste-free process for continuous flow enzymatic esterification using a double pervaporation system", Green. Chem., vol. 10, pp. 1284-1287, 2008.

[20] S.-H. Chen, Q. Zhao, and X.-W. Xu, "Preparation and characterization of a novel benzimidazolium bronsted acidic ionic liquid and its application in esterifications", J. Chem. Sci., vol. 120, pp. 481-483, 2008.

[21] Y. Leng, J. Wang, D. Zhu, X. Ren, H. Ge, and L. Shen, "Heteropolyanion-based ionic liquids: reaction-induced selfseparation catalysts for esterification", Angew. Chem. Int. Ed. Engl., vol. 48, pp. 168-171, 2009.

[22] D. Naydenov, and H.-J. Bart, "Ternary liquid-liquid equilibria for six systems containing ethylacetate + ethanol or acetic acid + an imidazolium-based ionic liquid with a hydrogen sulfate anion at 313.2 K”, J. Chem. Eng. Data, vol.52, pp. 2375-2381, 2007.

[23] D. Naydenov, and H.-J. Bart, "Ternary liquid-liquid equilibria for systems containing alcohol or acetic acid + ester + 1-ethyl-3- methylimidazolium hydrogen sulfate at $313.2 \mathrm{~K}$ Using headspace gas chromatography", J. Chem. Eng. Data, vol. 54, pp. 43-47, 2009.

[24] E.E. Reid, M. Peterson, and J. Way, "Esterification" In Unit Processes in Organic Synthesis, P. H. Groggings, Ed. Kōgakusha, McGraw-Hill, 1958, pp. 694-749.

[25] S. I. Kirbaslar, Z. Baykal, and U. Dramur, "Esterification of acetic acid with ethanol catalysed by an acidic ion-exchange resin", Turk. J. Eng. Environ. Sci., vol. 25, pp. 569-577, 2001.

[26] W. J. Jones, and A. Lapworth, "Equilibrium in the system: ethyl alcohol, acetic acid, ethyl acetate and water, and its apparent displacement by hydrogen chloride", J. Chem. Soc. Trans., vol. 99, pp. 1427-1432, 1911.

[27] Y. T. Tang, H.-P. Huang, and I-L. Chien, "Design of a complete ethyl acetate reactive distillation system", J. Chem. Eng. Jpn., vol. 36, pp. 1352-1363, 2003.

[28] W. Kaltenbrunner, "Kinetik der durch das Ionenaustauscherharz Dowex Monosphere $650 \mathrm{C}$ katalysierten Veresterungsreaktion von Essigsäure mit n-Propanol im diskontinuierlichen Rührkessel“, Diploma thesis, Linz, Austria, University of Linz, 1994.

[29] L. A. Blanchard, and J. F. Brennecke, "Esterification of acetic acid with ethanol in carbon dioxide", Green Chem., vol. 3, pp. 17-19, 2001.

[30] D. Naydenov, and H.-J. Bart, "Untersuchungen zur Reaktivextraktion mit ionischen Flüssigkeiten", Chem. Ing. Technik, vol. 80, pp. 137-143, 2008.

Received: February 26, 2009

Revised: March 06, 2009

Accepted: April 07, 2009

(C) Naydenovet al.; Licensee Bentham Open.

This is an open access article licensed under the terms of the Creative Commons Attribution Non-Commercial License (http://creativecommons.org/licenses/by-nc/3.0/) which permits unrestricted, non-commercial use, distribution and reproduction in any medium, provided the work is properly cited. 